\title{
Biomassa e nutrientes de eucalipto cultivado em sistema agrossilvipastoril
}

\author{
Charlote WINK $^{1 *}$, Anderson LANGE ${ }^{1}$, Kamile Zompero ARAÚJO ${ }^{1}$, Ana Paula Silveira de ALMEIDA ${ }^{1}$, \\ Maurel BEHLING², Flávio Jesus WRUCK ${ }^{2}$ \\ ${ }^{1}$ Instituto de Ciências Agrárias e Ambientais, Universidade Federal de Mato Grosso, Sinop, Mato Grosso, Brasil. \\ ${ }^{2}$ Embrapa Agrossilvipastoril, Sinop, Mato Grosso, Brasil. \\ *E-mail: charlote.wink@gmail.com
}

Recebido em dezembro/2017; Aceito em maio/2018.

\begin{abstract}
RESUMO: O objetivo foi quantificar a biomassa e o estoque de nutrientes do híbrido Eucalyptus grandis Hill ex Maiden x E. urophylla S. T. Blake (clone H13) aos 60 meses em sistema agrossilvipastoril. O estudo da biomassa residual (folhas e galhos finos), galhos grossos, madeira e casca e do estoque de nutrientes de eucalipto foi realizado na unidade de referência tecnológica de integração lavoura-pecuária-floresta em Nova Canaã do Norte, MT, cultivado em arranjo simples $(2 \times 20 \mathrm{~m})$, duplo $(3 \times 2 \times 20 \mathrm{~m})$, triplo $(3 \times 2 \times 20 \mathrm{~m})$ e em monocultivo $(4 \times 3 \mathrm{~m})$. Apenas a biomassa de galhos grossos, madeira e casca apresentaram diferenças significativas entre arranjos. Os nutrientes se acumularam em maior quantidade na madeira, e em ordem decrescente na casca, biomassa residual e nos galhos grossos. A biomassa residual, galhos grossos e casca correspondem a fração expressiva de nutrientes que permanece no sistema, e que contribuem para menor reposição na manutenção desses, após a colheita. O arranjo triplo apresentou maior acúmulo nutricional comparado ao arranjo simples e duplo. A eficiência nutricional é maior no arranjo de linha tripla devido a maior competição intraespecífica. $\mathrm{O}$ teor de nutrientes nas diferentes posições ao longo do fuste não apresentou variações nutricionais significativas para a madeira, diferentemente para a casca.
\end{abstract}

Palavras-chave: sistema iLPF, nutrição florestal, compartimentos.

\section{Biomass and nutrients of eucalyptus cultivated in agrossilvipastoral system}

\begin{abstract}
The objective was to quantify the biomass and nutrient stock of the hybrid Eucalyptus grandis Hill ex Maiden x E. urophylla S. T. Blake, (clone H13) at 60 months in the agrosilvipastoril system. The study of the residual biomass (leaves and thin branches), thick branches, wood and bark and of the nutrient stock of eucalyptus was carried out in the technological unit of crop-livestock-forest integration in Nova Canaã do Norte, MT, cultivated in a simple arrangement $(2 \times 20 \mathrm{~m})$, double $(3 \times 2 \times 20 \mathrm{~m})$, triple $(3 \times 2 \times 20 \mathrm{~m})$ and monoculture $(4 \times 3 \mathrm{~m})$. Only the biomass of thick branches, wood and bark presented significant differences between arrangements. The nutrients accumulate in greater quantity in the wood, and in descending order in the bark, residual biomass and in the thick branches. Residual biomass, thick branches and bark correspond to the expressive fraction of nutrients that remain in the system, and which contribute to a lower replacement in the maintenance of these, after harvesting. The triple arrangement presented greater nutritional accumulation compared to the single and double arrangement. The nutritional efficiency is higher in the triple line arrangement due to greater intraspecific competition. The nutrient content in the different positions along the stem did not present significant nutritional variations for the wood, differently for the bark.
\end{abstract}

Keywords: CLF integration system, forest nutrition, compartments.

\section{INTRODUÇÃO}

Os sistemas agroflorestais (SAF) compreendem o consórcio de espécies arbóreas lenhosas, agrícolas e/ou animais, representado por diferentes modalidades, incluindo, de forma generalizada, o sistema de integração lavourapecuária-floresta (iLPF). Essa tecnologia de produção integrada apresenta atualmente uma expansão em área produtiva no país, com expectativa de crescimento para os próximos anos.

Essa expansão é motivada pela importância dos sistemas de produção integrada em ser uma alternativa viável de uso do solo pois proporciona o melhor aproveitamento da área e diversifica a renda do produtor. Esses sistemas integrados reduzem o uso de fertilizantes e insumos (BALBINO et al., 2011), além de recuperar áreas improdutivas ou de baixo potencial.
Das espécies arbóreas mais utilizadas na produção integrada e com bons resultados de crescimento (CARVALHO, 2017) está o híbrido Eucalyptus grandis x E. urophylla (clone $\mathrm{H} 13)$. O rápido crescimento das espécies e híbridos de eucalipto ocorre por sua adaptação as variadas condições edafoclimáticas, constituindo em uma matéria prima importante para diferentes segmentos do mercado florestal.

$\mathrm{O}$ crescimento das árvores e a consequente produção de biomassa e o acúmulo de nutrientes são influenciados pelo sítio e pelos aspectos do plantio. Para o sítio considera-se as características do solo, envolvendo especialmente a fertilidade natural, já em relação ao plantio, considera-se a qualidade da muda ou do genótipo, o preparo do solo, a correta adubação, além da orientação das faixas de plantio das árvores no consórcio, influenciada essa pela incidência solar. 
Desse modo, a biomassa florestal compreende todo o material vegetal segmentado nos diferentes compartimentos (folhas, galhos, casca, madeira e raiz) das árvores, depositado ou devolvido ao sítio, naturalmente ou após intervenção antrópica. Já o resíduo é todo o compartimento da biomassa que permanece após a colheita florestal, como as folhas e em alguns casos, os galhos grossos e finos e a casca.

A quantificação do resíduo permite avaliar o potencial de uso e aproveitamento da área, uma vez que diversos estudos apontam os benefícios desse material, restritos são os resultados ao que se refere a produção de resíduos, bem como da produção de biomassa em sistemas de produção consorciada.

A devolução de biomassa e a deposição de serapilheira, bem como a correta adubação abrangem os mecanismos de devolução de nutrientes ao sistema, completando o ciclo nutricional, sendo fundamental ao crescimento, incremento e rendimento final do plantio ou da cultura. Por outro lado, a exportação nutricional devido a colheita das árvores influencia na produtividade, uma vez que ela está associada a exportação nutricional do sistema, influenciando assim no equilíbrio do balanço dos nutrientes no ciclo biogeoquímico.

Assim, o conhecimento da importação e exportação de nutrientes pelos diferentes compartimentos das árvores durante o seu ciclo de produção se torna importante para tomada de decisão quanto ações corretas de manejo ao nível das árvores e ao nível de sistema.

A grandeza dos teores nos compartimentos é variável, com as folhas concentrando a maior atividade metabólica, comparado ao lenho, que é menor, e onde são alocados os nutrientes remobilizados e redistribuídos (BAGGIO; CARPANEZZI, 1997). Embora a madeira seja o compartimento com menor teor nutricional, a maior parte da biomassa se concentra nesse compartimento, com expressiva exportação de nutrientes, no entanto a variação na quantidade exportada é resultado da biomassa extraída. As diferenças no teor de nutrientes entre os compartimentos e no mesmo compartimento aumentam com a idade, em decorrência da translocação dos nutrientes de tecidos senescentes para regiões com maior atividade metabólica (REIS; BARROS, 1990). Portanto, a idade do tecido vegetal e consequentemente a mobilidade dos elementos explica a variação do teor dos nutrientes ao longo do tronco (RUBILAR et al., 2005).

A casca é rica em $\mathrm{Ca}, \mathrm{K}$ e $\mathrm{Mg}$, e esse alto teor de nutrientes é resultado das células de parênquima com cristais de oxalato e carbonato de $\mathrm{Ca}$ e $\mathrm{Mg}$. Esses minerais são importantes na sustentabilidade do sítio, uma vez que sua exportação pela colheita sem reposição, promove o empobrecimento gradual do solo com prejuízos na produtividade das florestas (FOELKEL, 2006).

O K é móvel nos tecidos da planta, concentrando-se nas áreas ativas de crescimento e está associado a processos vitais da planta (MARSCHNER, 1995). Consequentemente em plantios puros, pela maior competição devido a densidade, o componente arbóreo concentra o crescimento em altura, com menor produção de copa.

Portanto, o objetivo do estudo foi quantificar a biomassa e o estoque de nutrientes de Eucalyptus grandis x E. urophylla (clone H13) cultivado em diferentes arranjos de plantio de um sistema agrossilvipastoril.

\section{MATERIAL E MÉTODOS}

O estudo foi realizado em um sistema de integração lavoura-pecuária-floresta (iLPF) com 60 meses, cultivado em Nova Canaã do Norte-MT, localizado a $10^{\circ} 24^{\prime} 09,15^{\prime \prime} \mathrm{S}$ e 5543'22,88' O.

Conforme Köppen, o clima local é do tipo tropical chuvoso com estação seca (Am monsoon), com temperatura média de $25^{\circ} \mathrm{C}$ e precipitação média anual de 2.500 a 2.800 mm (ALVARES et al., 2014). O solo é classificado como Latossolo Vermelho Amarelo distroférrico de textura média (SANTOS et al., 2013).

Após a supressão da vegetação de floresta nativa, foi realizado o cultivo de pastagem forrageira da espécie Brachiaria brizantha cv Marandu por dois anos. Posteriormente, a área foi cultivada com culturas anuais por seis anos consecutivos, sendo dois deles no cultivo de arroz e o restante no cultivo de soja com cultivo de milho na safrinha. Sequencialmente, a área foi cultivada novamente com a forrageira e com pastejo de bovinos. Em janeiro de 2009, a pastagem foi dessecada com glifosato (2L.ha-1, 720 g.ha- ${ }^{-1}$ ), a cultura do arroz foi implantada com o plantio das árvores de eucalipto no sistema ILPF, envolvendo o preparo por cultivo mínimo. Nos primeiros dois anos da implantação, o eucalipto foi cultivado em consórcio com lavoura, e posteriormente com pastagem de Brachiaria brizantha cv Marandu.

As faixas de cultivo do eucalipto (E. grandis $x \quad E$. urophylla, clone H13) foram plantadas no sentido lestenordeste a oeste-sudoeste em talhões de 5 ha $(200 \times 250 \mathrm{~m})$, em arranjo de linha simples $(2 \times 20 \mathrm{~m})$, dupla $(3 \times 2 \times 20 \mathrm{~m})$, tripla $(3 \times 2 \times 20 \mathrm{~m})$ e em monocultivo $(4 \times 3 \mathrm{~m})$, representando uma densidade arbórea de 250, 435, 537 e 833 árvores por hectare, respectivamente nos arranjos de plantio.

No plantio das mudas, foram aplicados por planta, $200 \mathrm{~g}$ de NPK na formulação 01-18-18. Já aos 10 a 14 meses, realizou-se a adubação de cobertura com NPK na formulação de 20-00-10, sendo 100 g por planta, acrescido de $2 \%$ de boro e $1 \%$ de zinco.

Aos 60 meses, o diâmetro a altura do peito (dap) e a altura total média (ht), respectivamente, eram de $23,2 \mathrm{~cm}$ e $23,1 \mathrm{~m}$ no arranjo simples, $19,8 \mathrm{~cm}$ e $23,9 \mathrm{~m}$ no arranjo duplo, $19,6 \mathrm{~cm}$ e $24,7 \mathrm{~m}$ no triplo comparados a $17,8 \mathrm{~cm}$ e $25,9 \mathrm{~m}$ no monocultivo de eucalipto. Já o incremento médio anual era de $20,76 \mathrm{~m}^{3} \cdot \mathrm{ha}^{-1}$. ano no arranjo simples, $29,66 \mathrm{~m}^{3} \cdot \mathrm{ha}^{-1}$. ano no arranjo duplo, $39,72 \mathrm{~m}^{3}$.ha- ${ }^{-1}$ ano no triplo, comparado ao monocultivo que apresentava $39,20 \mathrm{~m}^{3} \cdot \mathrm{ha}^{-1}$.ano.

A biomassa foi quantificada em quatro árvores no arranjo de linha simples e no monocultivo, oito árvores no arranjo de linha dupla e doze árvores no arranjo de linha tripla. Para a escolha das árvores a serem abatidas considerou aquelas próximas ao ponto de coleta de solo em cada arranjo. Além disso, nessa escolha, observou-se também proporcionalmente as faces de exposição norte, sul e central, unicamente para o arranjo de linha dupla e tripla. Selecionadas as árvores, realizou-se o abate e a cubagem com e sem casca pelo método de Smalian, nas posições $0,1,3 \mathrm{~m}$ e a cada dois metros até a altura total com um diâmetro mínimo de $8,0 \mathrm{~cm}$. O fuste com diâmetro inferior a $8,0 \mathrm{~cm}$ foi descartado como ponteira.

A biomassa por árvore foi separada e pesada nos compartimentos residual (folhas e galhos finos), galhos grossos com diâmetro mínimo de $0,06 \mathrm{~m}$ (uso para energia na secagem de grãos), madeira e casca. Amostras da biomassa 
residual e dos galhos grossos foram secas em estufa a $65^{\circ} \mathrm{C}$ por 72 horas para determinação do peso seco total desses compartimentos. O peso seco total de madeira e casca foi determinado pelo produto do volume obtido na cubagem pela densidade básica desses compartimentos, obtidos a partir de discos com espessura de $3,0 \mathrm{~cm}$, retirados da base, 50 e $100 \%$ da altura total da árvore.

Amostras dos compartimentos, inclusive dos discos de madeira com casca obtidos das diferentes posições do fuste foram processadas em moinho de faca e avaliadas quanto ao teor de N, P, K, Ca, Mg, S, B e Zn (SILVA, 2009).

Em cada arranjo de plantio, a biomassa por hectare de cada compartimento foi determinada pelo produto do peso seco pela densidade de árvores por hectare. Já o estoque de nutrientes por hectare de cada compartimento foi obtido pelo produto do peso seco por hectare pelo teor do respectivo nutriente.

A comparação dos resultados da biomassa, teor e do estoque de nutrientes nos arranjos e na posição do tronco foi realizada por meio do teste não paramétrico de KruskallWallis a $10 \%$ de probabilidade.

\section{RESULTADOS}

A biomassa média total, independentemente do sistema, foi de $123,11 \mathrm{t} \mathrm{ha}^{-1}$, sendo $74,84 \%$ de madeira, $12,73 \%$ de casca, $6,93 \%$ de galhos grossos e 5,5\% de biomassa residual. Desse total médio, 141,16 t ha-1 ocorreu no arranjo triplo, $106,32 \mathrm{t} \mathrm{ha}^{-1}$ no arranjo duplo, 73,84 $\mathrm{t} \mathrm{ha}^{-1}$ ocorreu no arranjo simples, comparado aos $171,11 \mathrm{t} \mathrm{ha}^{-1}$ no monocultivo de eucalipto (Figura 1).

A biomassa de madeira, da casca e o total acumulado no monocultivo superou a produção registrada nos demais arranjos, e esses foram significativamente diferentes no monocultivo quando comparado ao arranjo simples.

Para a biomassa residual (folhas e galhos finos), o arranjo duplo e triplo apresentaram maior acúmulo quando comparado ao arranjo simples e ao monocultivo de eucalipto, mas não diferiram entre si. Para a biomassa de galhos grossos, o arranjo duplo e triplo apresentaram valores significativamente inferiores ao monocultivo, o qual não diferiu do arranjo simples.

Biomassa de $E$. grandis Hill ex Maiden x $E$. urophylla $\mathbf{S}$. T. Blake (clone H13) em sistema agrossilvipastoril
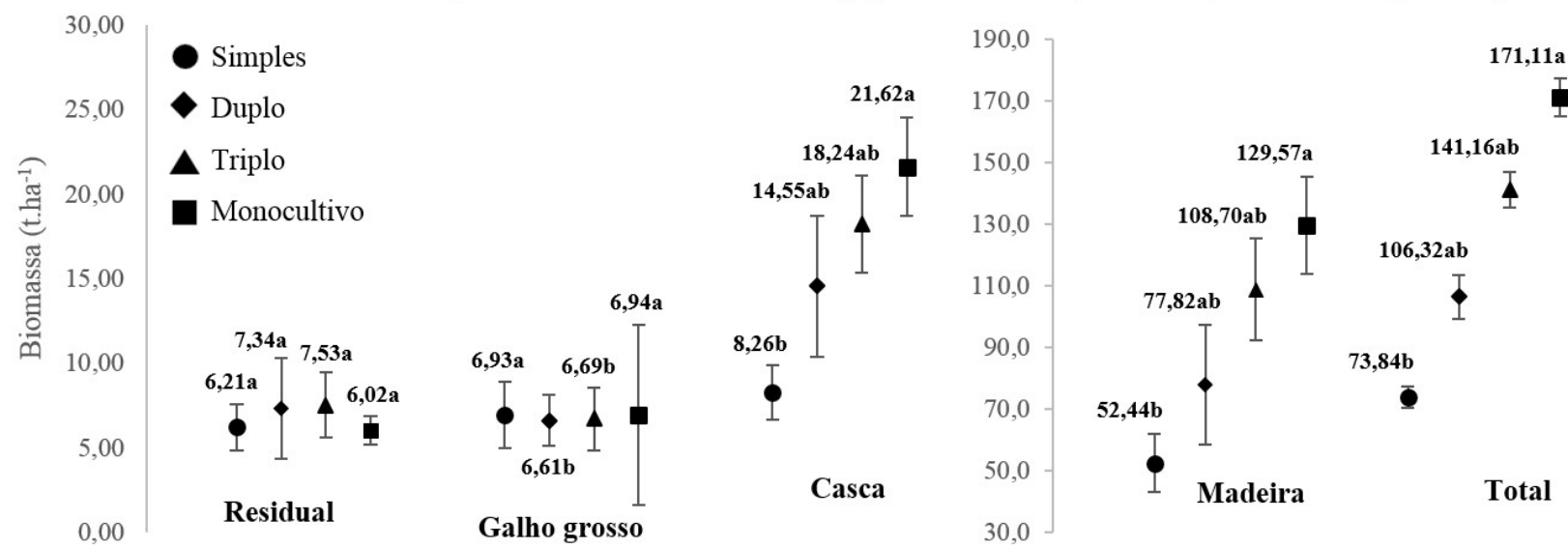

Figura 1. Biomassa ( $\mathrm{tha}^{-1}$ ) residual (folhas e galhos finos), galhos grossos, madeira e casca de E. grandis x E. urophylla (clone H13), cultivado em arranjo de linha simples, dupla, tripla em sistema agrossilvipastoril e no monocultivo. Médias seguidas pela mesma letra não diferem significativamente entre arranjos espaciais para cada compartimento avaliado pelo Teste de Kruskal-Wallis ao nível de 5\% de probabilidade. Barras verticais indicam o desvio padrão

Figure 1. Residual (leaves and fine branches), thick branches, wood and bark biomass ( $\mathrm{t} \mathrm{ha}^{-1}$ ) of E. grandis x E. urophylla (clone H13), cultivated in single, double, triple arrangement in agrossilvipastoral systems and monoculture. Means followed by the same letter did not differ significantly between spatial arrangements for each compartment evaluated by the Kruskal-Wallis test at the $5 \%$ probability level. Vertical bars indicate the standard deviation.

Em relação a partição nutricional, os teores de $\mathrm{N}$ foram influenciados na madeira e no total acumulado, já os teores de $\mathrm{P}$ foram influenciados em todos os compartimentos, exceto na casca. Os teores de $\mathrm{Ca}, \mathrm{Mg}$ e $\mathrm{B}$ diferiram na madeira, na biomassa residual e nos galhos grossos, respectivamente (Tabela 1). Destaca-se que a biomassa residual, especialmente em função da presença de folhas e material tenro, tem maiores teores de $\mathrm{N}, \mathrm{P}, \mathrm{K}$ e $\mathrm{Mg}$ que outros compartimentos, já a casca apresentou alto teor de $\mathrm{Ca}$. No caso dos micronutrientes, o teor de B apresenta valores altos, independente do compartimento da biomassa avaliado. Os teores de $\mathrm{K}, \mathrm{Ca}, \mathrm{Mg}, \mathrm{S}, \mathrm{B}$ e $\mathrm{Zn}$ apresentaram diferenças mínimas entres os sistemas quanto aos compartimentos avaliados.

$\mathrm{O}$ estoque médio por compartimento da biomassa nos diferentes arranjos de plantio pode ser observado na Tabela 2.
Comparando os sistemas entre si, observou-se estoques de nutrientes significativos somente na madeira, casca e galhos grossos. Independentemente do compartimento e do arranjo de plantio, o estoque de nutrientes total médio foi de 533,81 kg.ha- ${ }^{-1}$ para o N, 72,94 kg.ha-1 para o P, 260,19 kg.ha-1 para o $\mathrm{K}, 1236,82 \mathrm{~kg} \mathrm{ha}^{-1}$ para o Ca, 241,62 kg.ha-1 para o Mg, 72,95 kg.ha-1 para o S, 4050,51 g.ha-1 para o B e de 977,84 g.ha-1 para o $\mathrm{Zn}$.

O monocultivo de eucalipto registrou os maiores estoques de nutrientes nos diferentes compartimentos, todos significativos quando comparados aos demais arranjos. Em sequência, em ordem decrescente dos estoques aparecem o arranjo triplo, duplo e simples. Quando comparado apenas os arranjos simples, duplo e triplo, pode-se afirmar que os estoques nutricionais não diferem entre si, exceto para o $\mathrm{P}$ e o $\mathrm{Mg}$ na madeira e para o $\mathrm{Ca}, \mathrm{S}$ e o $\mathrm{Zn}$ na casca (Tabela 2). 
Tabela 1. Teor médio de nutrientes $\left(\mathrm{g} \mathrm{kg}^{-1} \mathrm{e} \mathrm{mg} \mathrm{kg}^{-1}\right)$ nos compartimentos da biomassa do eucalipto (clone H13) nos diferentes arranjos do sistema agrossilvipastoril, em Nova Canãa do Norte, MT.

Table 1. Mean nutrient content $\left(\mathrm{g} \mathrm{kg}^{-1}\right.$ and $\left.\mathrm{mg} \mathrm{kg}^{-1}\right)$ in the biomass compartments of eucalyptus (clone $\left.\mathrm{H} 13\right)$ in the different arrangements of the agrossilvipastoral system, in Nova Canãa do Norte, MT.

\begin{tabular}{|c|c|c|c|c|c|c|c|c|c|}
\hline & & \multicolumn{5}{|c|}{ Macronutrientes $\left(\mathrm{g} \mathrm{kg}^{-1}\right)$} & \multicolumn{3}{|c|}{ Micronutrientes $\left(\mathrm{mg} \mathrm{kg}^{-1}\right)$} \\
\hline & & $\mathrm{N}$ & $\mathrm{P}$ & $\mathrm{K}$ & $\mathrm{Ca}$ & $\mathrm{Mg}$ & $\mathrm{S}$ & $\mathrm{B}$ & $\mathrm{Zn}$ \\
\hline \multirow{4}{*}{$\begin{array}{c}\text { Biomassa } \\
\text { residual } \\
(\mathrm{F}+\mathrm{GF})\end{array}$} & Simples & $18,16 \mathrm{a}$ & $1,13 \mathrm{a}$ & $10,13 \mathrm{a}$ & $11,81 \mathrm{a}$ & $3,70 \mathrm{~b}$ & $0,67 \mathrm{a}$ & $32,90 \mathrm{a}$ & $15,45 \mathrm{a}$ \\
\hline & Duplo & $20,33 \mathrm{a}$ & $1,04 \mathrm{ab}$ & $10,34 \mathrm{a}$ & $12,59 \mathrm{a}$ & $4,67 \mathrm{ab}$ & $0,64 \mathrm{a}$ & $32,91 \mathrm{a}$ & $16,73 \mathrm{a}$ \\
\hline & Tripla & $18,04 \mathrm{a}$ & $0,93 \mathrm{~b}$ & $10,93 \mathrm{a}$ & $11,13 \mathrm{a}$ & $3,71 \mathrm{~b}$ & $0,69 \mathrm{a}$ & $32,92 \mathrm{a}$ & $14,83 \mathrm{a}$ \\
\hline & Monocultivo & $16,15 \mathrm{a}$ & $1,08 \mathrm{ab}$ & $10,68 \mathrm{a}$ & $12,77 \mathrm{a}$ & 5,08 a & $0,61 \mathrm{a}$ & $32,91 \mathrm{a}$ & $17,13 \mathrm{a}$ \\
\hline \multirow{4}{*}{$\begin{array}{l}\text { Galhos } \\
\text { grossos }\end{array}$} & Simples & $2,99 \mathrm{a}$ & $0,67 \mathrm{ab}$ & $2,11 \mathrm{a}$ & $10,59 \mathrm{a}$ & $2,07 \mathrm{a}$ & $0,46 \mathrm{a}$ & $32,92 \mathrm{a}$ & $8,50 \mathrm{a}$ \\
\hline & Duplo & $2,59 \mathrm{a}$ & $0,76 \mathrm{ab}$ & $2,11 \mathrm{a}$ & $10,56 \mathrm{a}$ & $2,01 \mathrm{a}$ & $0,56 \mathrm{a}$ & $32,91 \mathrm{ab}$ & $7,33 \mathrm{a}$ \\
\hline & Tripla & $3,01 \mathrm{a}$ & $0,62 \mathrm{~b}$ & $1,99 \mathrm{a}$ & $9,91 \mathrm{a}$ & $2,17 \mathrm{a}$ & $0,59 \mathrm{a}$ & $32,91 \mathrm{a}$ & $7,36 \mathrm{a}$ \\
\hline & Monocultivo & $2,66 \mathrm{a}$ & $0,87 \mathrm{a}$ & $2,46 \mathrm{a}$ & $10,40 \mathrm{a}$ & $2,04 \mathrm{a}$ & $0,62 \mathrm{a}$ & $32,90 \mathrm{~b}$ & $7,44 \mathrm{a}$ \\
\hline \multirow{4}{*}{ Madeira } & Simples & $3,50 a b$ & $0,34 \mathrm{~b}$ & $0,70 \mathrm{a}$ & $7,03 \mathrm{ab}$ & $1,26 \mathrm{a}$ & $0,66 \mathrm{a}$ & $32,90 \mathrm{a}$ & $5,86 \mathrm{a}$ \\
\hline & Duplo & $3,44 \mathrm{~b}$ & $0,61 \mathrm{a}$ & $0,76 \mathrm{a}$ & $6,86 \mathrm{~b}$ & $1,31 \mathrm{a}$ & $0,57 \mathrm{a}$ & $32,90 \mathrm{a}$ & $5,28 \mathrm{a}$ \\
\hline & Tripla & $3,54 \mathrm{ab}$ & $0,46 \mathrm{ab}$ & $0,68 \mathrm{a}$ & $7,18 \mathrm{ab}$ & $1,55 \mathrm{a}$ & $0,59 \mathrm{a}$ & $32,90 \mathrm{a}$ & $5,67 \mathrm{a}$ \\
\hline & Monocultivo & $3,67 \mathrm{a}$ & $0,31 \mathrm{~b}$ & $0,73 \mathrm{a}$ & $7,61 \mathrm{a}$ & $1,54 \mathrm{a}$ & $0,51 \mathrm{a}$ & $32,90 \mathrm{a}$ & $6,53 \mathrm{a}$ \\
\hline \multirow{4}{*}{ Casca } & Simples & $3,71 \mathrm{a}$ & $1,22 \mathrm{a}$ & $5,48 \mathrm{a}$ & $25,17 \mathrm{a}$ & $3,60 \mathrm{a}$ & $0,74 \mathrm{a}$ & $32,91 \mathrm{a}$ & $16,30 \mathrm{a}$ \\
\hline & Duplo & $3,68 \mathrm{a}$ & $1,24 \mathrm{a}$ & $6,10 \mathrm{a}$ & $26,02 \mathrm{a}$ & $4,00 \mathrm{a}$ & $0,76 \mathrm{a}$ & $32,91 \mathrm{a}$ & $16,16 \mathrm{a}$ \\
\hline & Tripla & $3,71 \mathrm{a}$ & $1,28 \mathrm{a}$ & $6,78 \mathrm{a}$ & $24,98 \mathrm{a}$ & $3,94 \mathrm{a}$ & $0,70 \mathrm{a}$ & $32,90 \mathrm{a}$ & $17,03 \mathrm{a}$ \\
\hline & Monocultivo & $3,78 \mathrm{a}$ & $1,32 \mathrm{a}$ & $7,08 \mathrm{a}$ & $25,73 \mathrm{a}$ & $3,89 \mathrm{a}$ & $0,60 \mathrm{a}$ & $32,90 \mathrm{a}$ & $16,99 \mathrm{a}$ \\
\hline \multirow{4}{*}{ Total } & Simples & $4,70 \mathrm{a}$ & $0,54 \mathrm{~b}$ & $2,16 \mathrm{a}$ & 9,79 a & $1,81 \mathrm{a}$ & $0,65 \mathrm{a}$ & $32,91 \mathrm{a}$ & $8,08 \mathrm{a}$ \\
\hline & Duplo & $4,67 \mathrm{ab}$ & $0,74 \mathrm{a}$ & $2,25 \mathrm{a}$ & $10,13 \mathrm{a}$ & $2,00 \mathrm{a}$ & $0,61 \mathrm{a}$ & $32,90 \mathrm{a}$ & $7,65 \mathrm{a}$ \\
\hline & Tripla & $4,28 \mathrm{ab}$ & $0,60 \mathrm{ab}$ & $2,10 \mathrm{a}$ & $9,77 \mathrm{a}$ & $1,97 \mathrm{a}$ & $0,61 \mathrm{a}$ & $32,90 \mathrm{a}$ & $7,71 \mathrm{a}$ \\
\hline & Monocultivo & $4,05 \mathrm{~b}$ & $0,51 \mathrm{~b}$ & $2,04 \mathrm{a}$ & $10,41 \mathrm{a}$ & $2,01 \mathrm{a}$ & $0,53 \mathrm{a}$ & $32,90 \mathrm{a}$ & $8,37 \mathrm{a}$ \\
\hline
\end{tabular}

onde F:GF: folhas e galhos finos; N: nitrogênio; P: fósforo; K: potássio; Ca: cálcio; Mg: magnésio; S: enxofre; B: boro; Zn: zinco; médias seguidas pela mesma letra na coluna, não diferem significativamente pelo Teste de Kruskall-Wallis ao nível de 5\% de probabilidade.

Tabela 2. Estoque médio de nutrientes $\left(\mathrm{kg} \mathrm{ha}^{-1} \mathrm{e} \mathrm{g} \mathrm{ha}^{-1}\right)$ nos compartimentos da biomassa do eucalipto (clone H13) nos diferentes arranjos do sistema agrossilvipastoril, em Nova Canãa do Norte, MT.

Table 2. Mean nutrient stock $\left(\mathrm{kg} \mathrm{ha}^{-1}\right.$ and $\left.\mathrm{g} \mathrm{ha}^{-1}\right)$ in the biomass compartments of eucalyptus (clone $\mathrm{H} 13$ ) in the different arrangements of the agrossilvipastoral system in Nova Canãa do Norte, MT.

\begin{tabular}{|c|c|c|c|c|c|c|c|c|c|}
\hline \multirow[b]{2}{*}{ Compartimento } & \multirow[b]{2}{*}{ Arranjo } & \multicolumn{5}{|c|}{ Macronutrientes $\left(\mathrm{kg} \mathrm{ha}^{-1}\right)$} & \multicolumn{3}{|c|}{ Micronutrientes $\left(\mathrm{g} \mathrm{ha}^{-1}\right)$} \\
\hline & & $\mathrm{N}$ & $\mathrm{P}$ & $\mathrm{K}$ & $\mathrm{Ca}$ & $\mathrm{Mg}$ & $\mathrm{S}$ & $\mathrm{B}$ & $\mathrm{Zn}$ \\
\hline \multirow{4}{*}{$\begin{array}{c}\text { Biomassa } \\
\text { residual }(\mathrm{F}+\mathrm{GF})\end{array}$} & Simples & $111,9 \mathrm{a}$ & $7,0 \mathrm{a}$ & $64,1 \mathrm{a}$ & $74,6 \mathrm{a}$ & $23,2 \mathrm{a}$ & $4,3 \mathrm{a}$ & $204,4 \mathrm{a}$ & $97,8 \mathrm{a}$ \\
\hline & Duplo & $150,4 \mathrm{a}$ & $7,6 \mathrm{a}$ & $74,2 \mathrm{a}$ & $94,1 \mathrm{a}$ & $36,5 \mathrm{a}$ & $4,3 \mathrm{a}$ & $241,5 \mathrm{a}$ & $125,1 \mathrm{a}$ \\
\hline & Tripla & $134,4 \mathrm{a}$ & $7,0 \mathrm{a}$ & 83,3 a & 83,6 a & $27,4 \mathrm{a}$ & $5,1 \mathrm{a}$ & $247,8 \mathrm{a}$ & $110,1 \mathrm{a}$ \\
\hline & Monocultivo & $98,8 \mathrm{a}$ & $6,5 \mathrm{a}$ & $64,2 \mathrm{a}$ & $76,3 \mathrm{a}$ & $30,7 \mathrm{a}$ & $3,0 \mathrm{a}$ & $198,1 \mathrm{a}$ & $103,4 \mathrm{a}$ \\
\hline \multirow{4}{*}{ Galhos grossos } & Simples & $21,2 \mathrm{ab}$ & $4,7 \mathrm{ab}$ & $14,8 \mathrm{a}$ & $73,0 \mathrm{ab}$ & $14,5 \mathrm{a}$ & $3,1 \mathrm{~b}$ & $228,1 \mathrm{ab}$ & $57,9 \mathrm{ab}$ \\
\hline & Duplo & $17,1 \mathrm{~b}$ & $5,4 \mathrm{ab}$ & $13,9 \mathrm{a}$ & $69,0 \mathrm{ab}$ & $13,1 \mathrm{a}$ & $3,7 \mathrm{~b}$ & $217,6 \mathrm{~b}$ & $47,7 \mathrm{~b}$ \\
\hline & Tripla & $20,0 \mathrm{ab}$ & $4,2 \mathrm{~b}$ & $13,9 \mathrm{a}$ & $67,4 \mathrm{~b}$ & $14,6 \mathrm{a}$ & $3,9 \mathrm{ab}$ & $220,3 \mathrm{~b}$ & $50,1 \mathrm{ab}$ \\
\hline & Monocultivo & $37,3 \mathrm{a}$ & $11,3 \mathrm{a}$ & $34,6 \mathrm{a}$ & $143,2 \mathrm{a}$ & $28,5 \mathrm{a}$ & $8,6 \mathrm{a}$ & $457,1 \mathrm{a}$ & $104,0 \mathrm{a}$ \\
\hline \multirow{4}{*}{ Madeira } & Simples & $183,3 \mathrm{~b}$ & $18,1 \mathrm{~b}$ & $36,1 \mathrm{~b}$ & $368,6 \mathrm{~b}$ & $66,2 \mathrm{~b}$ & $34,5 \mathrm{~b}$ & $1725,7 \mathrm{~b}$ & $309,0 \mathrm{~b}$ \\
\hline & Duplo & $267,0 \mathrm{ab}$ & $48,7 \mathrm{a}$ & $60,3 \mathrm{ab}$ & $530,9 \mathrm{ab}$ & $101,3 \mathrm{ab}$ & $46,3 \mathrm{ab}$ & $2560,6 \mathrm{ab}$ & $404,8 \mathrm{~b}$ \\
\hline & Tripla & $383,6 \mathrm{ab}$ & $50,8 \mathrm{a}$ & $75,8 \mathrm{ab}$ & $776,2 \mathrm{ab}$ & $167,1 \mathrm{a}$ & $65,0 \mathrm{ab}$ & $3576,1 \mathrm{ab}$ & $613,6 \mathrm{ab}$ \\
\hline & Monocultivo & $476,0 \mathrm{a}$ & $40,7 \mathrm{ab}$ & $93,3 \mathrm{a}$ & $985,9 \mathrm{a}$ & $198,4 \mathrm{a}$ & $65,9 \mathrm{a}$ & $4262,7 \mathrm{a}$ & $842,6 \mathrm{a}$ \\
\hline \multirow{4}{*}{ Casca } & Simples & $30,7 \mathrm{~b}$ & $9,9 \mathrm{~b}$ & $45,2 \mathrm{~b}$ & $207,0 \mathrm{~b}$ & $29,9 \mathrm{~b}$ & $6,0 \mathrm{~b}$ & $271,7 \mathrm{~b}$ & $134,5 \mathrm{~b}$ \\
\hline & Duplo & $54,6 \mathrm{ab}$ & $18,3 \mathrm{ab}$ & $90,7 \mathrm{ab}$ & $378,2 \mathrm{ab}$ & $59,2 \mathrm{ab}$ & $11,3 \mathrm{ab}$ & $478,8 \mathrm{ab}$ & $227,6 \mathrm{ab}$ \\
\hline & Tripla & $67,6 \mathrm{ab}$ & $23,5 \mathrm{ab}$ & $122,0 \mathrm{ab}$ & $450,7 \mathrm{a}$ & $71,7 \mathrm{ab}$ & $13,0 \mathrm{a}$ & $660,1 \mathrm{ab}$ & $308,4 \mathrm{a}$ \\
\hline & Monocultivo & $81,5 \mathrm{a}$ & $28,1 \mathrm{a}$ & $154,5 \mathrm{a}$ & $568,7 \mathrm{a}$ & $84,4 \mathrm{a}$ & $13,2 \mathrm{a}$ & $711,3 \mathrm{a}$ & $374,8 \mathrm{a}$ \\
\hline \multirow{4}{*}{ Total } & Simples & $347,0 \mathrm{~b}$ & $39,7 \mathrm{~b}$ & $160,2 \mathrm{~b}$ & $723,1 \mathrm{~b}$ & $133,7 \mathrm{~b}$ & $48,0 \mathrm{~b}$ & $2429,9 \mathrm{~b}$ & $599,2 \mathrm{~b}$ \\
\hline & Duplo & $489,1 \mathrm{ab}$ & $80,0 \mathrm{ab}$ & $239,1 \mathrm{ab}$ & $1072,2 \mathrm{ab}$ & $210,0 \mathrm{ab}$ & $65,6 \mathrm{ab}$ & $3498,5 \mathrm{ab}$ & $805,2 \mathrm{~b}$ \\
\hline & Tripla & $605,6 \mathrm{a}$ & $85,5 \mathrm{ab}$ & $294,8 \mathrm{ab}$ & $1377,8 \mathrm{ab}$ & $280,8 \mathrm{ab}$ & $87,0 \mathrm{a}$ & $4644,3 \mathrm{ab}$ & $1082,3 \mathrm{ab}$ \\
\hline & Monocultivo & $693,6 \mathrm{a}$ & $86,5 \mathrm{a}$ & $346,6 \mathrm{a}$ & $1774,2 \mathrm{a}$ & $342,0 \mathrm{a}$ & $91,3 \mathrm{a}$ & $5629,3 \mathrm{a}$ & $1424,7 \mathrm{a}$ \\
\hline
\end{tabular}

onde F:GF: folhas e galhos finos; N: nitrogênio; P: fósforo; K: potássio; Ca: cálcio; Mg: magnésio; S: enxofre; B: boro; Zn: zinco; médias seguidas pela mesma letra na coluna, não diferem significativamente pelo Teste de Kruskall-Wallis ao nível de 5\% de probabilidade.

A madeira, em todos os arranjos, foi o compartimento que apresentou o maior estoque de nutrientes, com exceção para o $\mathrm{K}$, em que os maiores estoques ocorreram na casca e na biomassa residual. $\mathrm{O}$ estoque de nutrientes na madeira apresentou uma ordem de grandeza do $\mathrm{Ca}>\mathrm{N}>\mathrm{Mg}>\mathrm{K}>\mathrm{S}$ $>\mathrm{P}$ e $\mathrm{B}>\mathrm{Zn}$. Em seguida, com diminuição no estoque nutricional, tem-se a casca, a biomassa residual e os galhos grossos.
No geral, em termos percentuais, a biomassa residual (folhas e galhos finos) e a casca totalizam $34 \%$ do N, $37 \%$ do $\mathrm{P}, 67 \%$ do $\mathrm{K}, 39 \%$ do $\mathrm{Ca}, 38 \%$ do $\mathrm{Mg}, 21 \%$ do $\mathrm{S}, 18 \%$ B e $38 \%$ do $\mathrm{Zn}$, indicando a importância desses compartimentos na nutrição do sítio, uma vez que eles permanecem após a colheita (Figura 2). 


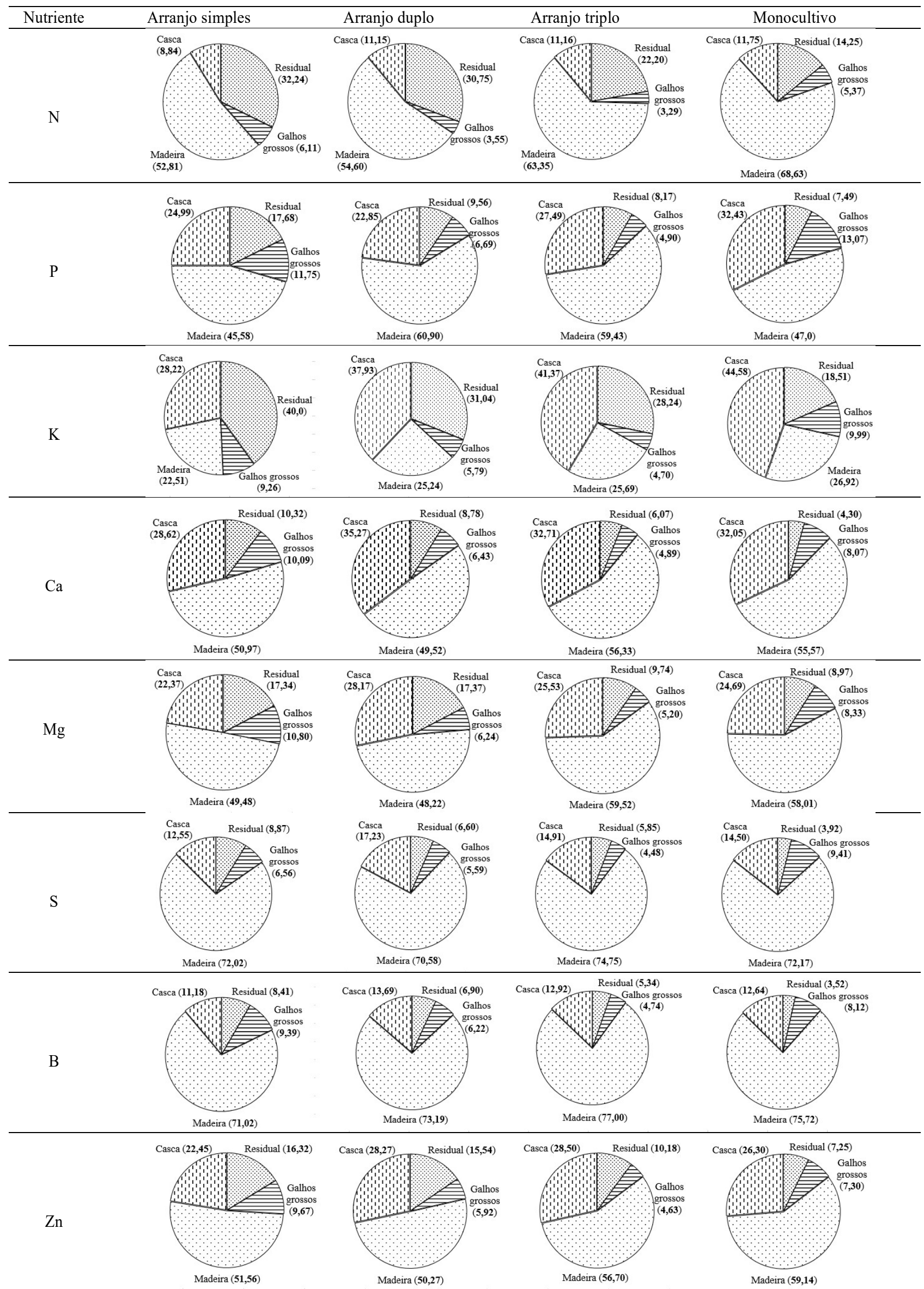

Figura 2. Percentual (\%) de distribuição do estoque de nutrientes nos compartimentos da biomassa do eucalipto (clone H13) nos diferentes arranjos do sistema agrossilvipastoril, em Nova Canaã do Norte, MT.

Figure 2. Percentage (\%) of nutrient stock distribution in the biomass compartments of eucalyptus (clone H13) in the different arrangements of the agrossilvipastoral system in Nova Canaã do Norte, MT. 
Ao comparar os extremos do arranjo simples em relação ao monocultivo, verifica-se que entre os macronutrientes, a sequência de exportação na madeira no arranjo simples é $\mathrm{S}>$ $\mathrm{N}>\mathrm{Ca}>\mathrm{Mg}>\mathrm{P}>\mathrm{K}$ e para os micronutrientes $\mathrm{B}>\mathrm{Zn}$. Já no monocultivo, a tendência é a mesma, apenas com inversão na ordem entre $\mathrm{Ca}$ e $\mathrm{Mg}$, demonstrando que o $\mathrm{S}$ é o macronutriente, em percentagem, mais exportado e o $\mathrm{K}$, o menor na madeira.

Em relação a variação nutricional ao longo do tronco das árvores, observou-se que o teor de nutrientes da madeira não apresentou diferença significativa quando comparando as posições dos discos obtidos da base, do meio e do ápice do tronco. Já o teor de nutrientes na casca apresentou diferença significativa para o N e P no arranjo simples e duplo, para o Zn no arranjo duplo e para o $\mathrm{Ca}$ e $\mathrm{Mg}$ nos três arranjos do ILPF, exceto para o monocultivo de eucalipto (Tabela 3).

Pode-se observar que entre os nutrientes, o $\mathrm{B}$ foi que mostrou valores constantes de teor nas diferentes posições do tronco em todos os arranjos, tanto para a madeira quanto para a casca.

Tabela 3. Teor médio de nutrientes $\left(\mathrm{g} \mathrm{kg}^{-1} \mathrm{e} \mathrm{mg} \mathrm{kg}^{-1}\right.$ ) nas diferentes posições do tronco das árvores de eucalipto (clone H13) cultivado nos diferentes arranjos do sistema agrossilvipastoril, em Nova Canãa do Norte, MT.

Table 3. Average nutrient content $\left(\mathrm{g} \mathrm{kg}^{-1}\right.$ and $\left.\mathrm{mg} \mathrm{kg}^{-1}\right)$ in the different positions of the stem of eucalyptus (clone H13) trees cultivate in the different arrangements of the agrossilvipastoral system, in Nova Canãa do Norte, MT.

\begin{tabular}{|c|c|c|c|c|c|c|c|c|c|}
\hline \multirow[b]{2}{*}{ Arranjo } & \multirow[b]{2}{*}{ Posição (\%) } & \multicolumn{5}{|c|}{ Macronutrientes $\left(\mathrm{g} \mathrm{kg}^{-1}\right)$} & \multicolumn{3}{|c|}{ Micronutrientes $\left(\mathrm{mg} \mathrm{kg}^{-1}\right)$} \\
\hline & & $\mathrm{N}$ & $\mathrm{P}$ & $\mathrm{K}$ & $\mathrm{Ca}$ & $\mathrm{Mg}$ & $\mathrm{S}$ & $\mathrm{B}$ & $\mathrm{Zn}$ \\
\hline \multicolumn{10}{|c|}{ Madeira } \\
\hline \multirow{3}{*}{ Simples } & 0 & $3,47 \mathrm{a}$ & $0,34 \mathrm{a}$ & $0,60 \mathrm{a}$ & $6,95 \mathrm{a}$ & $1,20 \mathrm{a}$ & $0,77 \mathrm{a}$ & $32,90 \mathrm{a}$ & $5,80 \mathrm{a}$ \\
\hline & 50 & $3,61 \mathrm{a}$ & $0,33 \mathrm{a}$ & $0,65 \mathrm{a}$ & $7,39 \mathrm{a}$ & $1,29 \mathrm{a}$ & $0,61 \mathrm{a}$ & $32,90 \mathrm{a}$ & $6,13 \mathrm{a}$ \\
\hline & 100 & $3,41 \mathrm{a}$ & $0,36 \mathrm{a}$ & $0,85 \mathrm{a}$ & $6,76 \mathrm{a}$ & $1,30 \mathrm{a}$ & $0,59 \mathrm{a}$ & $32,91 \mathrm{a}$ & $5,65 \mathrm{a}$ \\
\hline \multirow{3}{*}{ Duplo } & 0 & $3,42 \mathrm{a}$ & $0,53 \mathrm{a}$ & $0,55 \mathrm{a}$ & $6,80 \mathrm{a}$ & $1,18 \mathrm{a}$ & $0,52 \mathrm{a}$ & $32,90 \mathrm{a}$ & $4,86 \mathrm{a}$ \\
\hline & 50 & $3,43 \mathrm{a}$ & $0,63 \mathrm{a}$ & $0,64 \mathrm{a}$ & $6,82 \mathrm{a}$ & $1,44 \mathrm{a}$ & $0,60 \mathrm{a}$ & $32,91 \mathrm{a}$ & $5,86 \mathrm{a}$ \\
\hline & 100 & $3,47 \mathrm{a}$ & $0,68 \mathrm{a}$ & $1,10 \mathrm{a}$ & $6,96 \mathrm{a}$ & $1,32 \mathrm{a}$ & $0,60 \mathrm{a}$ & $32,91 \mathrm{a}$ & $5,10 \mathrm{a}$ \\
\hline \multirow{3}{*}{ Triplo } & 0 & $3,45 \mathrm{a}$ & $0,43 \mathrm{a}$ & $0,48 \mathrm{a}$ & $6,87 \mathrm{a}$ & $1,25 \mathrm{a}$ & $0,62 \mathrm{a}$ & $32,90 \mathrm{a}$ & $5,16 \mathrm{a}$ \\
\hline & 50 & $3,41 \mathrm{a}$ & $0,43 \mathrm{a}$ & $0,68 \mathrm{a}$ & $6,77 \mathrm{a}$ & $1,20 \mathrm{a}$ & $0,66 \mathrm{a}$ & $32,90 \mathrm{a}$ & $5,73 \mathrm{a}$ \\
\hline & 100 & $3,76 \mathrm{a}$ & $0,52 \mathrm{a}$ & $0,89 \mathrm{a}$ & 7,91 a & $2,22 \mathrm{a}$ & $0,49 \mathrm{a}$ & $32,90 \mathrm{a}$ & $6,13 \mathrm{a}$ \\
\hline \multirow{3}{*}{ Monocultivo } & 0 & $3,60 \mathrm{a}$ & $0,20 \mathrm{a}$ & $0,60 \mathrm{a}$ & $7,37 \mathrm{a}$ & $1,27 \mathrm{a}$ & $0,46 \mathrm{a}$ & $32,88 \mathrm{a}$ & $5,77 \mathrm{a}$ \\
\hline & 50 & $3,96 \mathrm{a}$ & $0,31 \mathrm{a}$ & $0,68 \mathrm{a}$ & $8,53 \mathrm{a}$ & $2,06 \mathrm{a}$ & $0,60 \mathrm{a}$ & $32,89 \mathrm{a}$ & $6,73 \mathrm{a}$ \\
\hline & 100 & $3,43 \mathrm{a}$ & $0,38 \mathrm{a}$ & $0,97 \mathrm{a}$ & $6,82 \mathrm{a}$ & $1,29 \mathrm{a}$ & $0,34 \mathrm{a}$ & $32,90 \mathrm{a}$ & $6,87 \mathrm{a}$ \\
\hline \multicolumn{10}{|c|}{ Casca } \\
\hline \multirow{3}{*}{ Simples } & 0 & $3,55 \mathrm{~b}$ & $0,43 \mathrm{~b}$ & $5,18 \mathrm{a}$ & $34,01 \mathrm{a}$ & $2,73 \mathrm{~b}$ & $0,71 \mathrm{a}$ & $32,91 \mathrm{a}$ & $14,68 \mathrm{a}$ \\
\hline & 50 & $3,54 \mathrm{~b}$ & $1,68 \mathrm{a}$ & $5,68 \mathrm{a}$ & $21,96 \mathrm{ab}$ & $3,61 \mathrm{ab}$ & $0,74 \mathrm{a}$ & $32,90 \mathrm{a}$ & $12,20 \mathrm{a}$ \\
\hline & 100 & $4,04 \mathrm{a}$ & $1,55 \mathrm{ab}$ & $5,58 \mathrm{a}$ & $19,53 \mathrm{~b}$ & $4,44 \mathrm{a}$ & $0,77 \mathrm{a}$ & $32,91 \mathrm{a}$ & $22,03 \mathrm{a}$ \\
\hline \multirow{3}{*}{ Duplo } & 0 & $3,34 \mathrm{~b}$ & $0,53 \mathrm{~b}$ & $5,33 \mathrm{a}$ & $35,34 \mathrm{a}$ & $2,69 \mathrm{~b}$ & $0,73 \mathrm{a}$ & $32,91 \mathrm{a}$ & $12,43 \mathrm{~b}$ \\
\hline & 50 & $3,51 \mathrm{~b}$ & $1,67 \mathrm{a}$ & $6,63 \mathrm{a}$ & $21,25 \mathrm{~b}$ & $3,78 \mathrm{a}$ & $0,72 \mathrm{a}$ & $32,91 \mathrm{a}$ & $13,74 \mathrm{ab}$ \\
\hline & 100 & $4,18 \mathrm{a}$ & $1,53 \mathrm{a}$ & $6,35 \mathrm{a}$ & $21,47 \mathrm{~b}$ & $5,55 \mathrm{a}$ & $0,83 \mathrm{a}$ & $32,91 \mathrm{a}$ & $22,33 \mathrm{a}$ \\
\hline \multirow{3}{*}{ Triplo } & 0 & $3,71 \mathrm{a}$ & $1,13 \mathrm{a}$ & $6,95 \mathrm{a}$ & $28,66 \mathrm{a}$ & $3,24 \mathrm{~b}$ & $0,62 \mathrm{a}$ & $32,90 \mathrm{a}$ & $15,96 \mathrm{a}$ \\
\hline & 50 & $3,64 \mathrm{a}$ & $1,51 \mathrm{a}$ & $6,46 \mathrm{a}$ & $21,22 \mathrm{ab}$ & $4,12 \mathrm{ab}$ & $0,76 \mathrm{a}$ & $32,91 \mathrm{a}$ & $16,99 \mathrm{a}$ \\
\hline & 100 & $3,76 \mathrm{a}$ & $1,20 \mathrm{a}$ & $6,93 \mathrm{a}$ & $25,05 \mathrm{a}$ & $4,45 \mathrm{a}$ & $0,71 \mathrm{a}$ & $32,91 \mathrm{a}$ & $18,13 \mathrm{a}$ \\
\hline \multirow{3}{*}{ Monocultivo } & 0 & $3,83 \mathrm{a}$ & $0,65 \mathrm{a}$ & $7,00 \mathrm{a}$ & $36,15 \mathrm{a}$ & $2,58 \mathrm{a}$ & $0,67 \mathrm{a}$ & $32,91 \mathrm{a}$ & $17,03 \mathrm{a}$ \\
\hline & 50 & $3,74 \mathrm{a}$ & $1,70 \mathrm{a}$ & $7,10 \mathrm{a}$ & $20,97 \mathrm{a}$ & $4,27 \mathrm{a}$ & $0,50 \mathrm{a}$ & $32,90 \mathrm{a}$ & $17,00 \mathrm{a}$ \\
\hline & 100 & $3,69 \mathrm{a}$ & $1,45 \mathrm{a}$ & $7,97 \mathrm{a}$ & $27,48 \mathrm{a}$ & $5,35 \mathrm{a}$ & $0,77 \mathrm{a}$ & $32,89 \mathrm{a}$ & $20,27 \mathrm{a}$ \\
\hline
\end{tabular}

onde N: nitrogênio; P: fósforo; K: potássio; Ca: cálcio; Mg: magnésio; S: enxofre; B: boro; Zn: zinco; médias seguidas pela mesma letra na coluna, não diferem significativamente pelo Teste de Kruskall-Wallis ao nível de 5\% de probabilidade.

\section{DISCUSSÃO}

A produção de biomassa arbórea registrada no sistema iLPF de arranjo duplo e triplo é similar ao registrado no monocultivo de eucalipto, excetuando apenas no arranjo de linha simples, no qual a produtividade foi menor.

Carvalho (2017) registrou um total de 76,34 Mg.ha-1 de biomassa total média em monocultivo de eucalipto (952

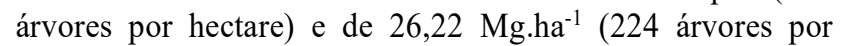
hectare) no sistema de iLPF. Já Coelho Junior (2015) registraram 144,3 $\mathrm{Mg} \mathrm{ha}^{-1}$ de biomassa residual (casca+folha+galhos), superior ao encontrado neste estudo de E. urograndis no sistema iLPF em espaçamento $3 \times 2 \mathrm{~m}$ do arranjo de linhas triplas.

As diferenças de biomassa registrada nos arranjos de plantio diferem de Müller et al. (2005), o qual afirma que os SAF produzem maior quantidade de biomassa de madeira, quando comparado ao monocultivo de eucalipto.
O estoque de biomassa na madeira no arranjo simples é inferior ao registrado para o mesmo híbrido aos 11 anos, em plantio agrossilvipastoril no espaçamento $10 \times 4 \mathrm{~m}$ (TSUKAMOTO-FILHO et al., 2004). Isso pode ser explicado pela diferença na idade, uma vez que com o aumento da mesma ocorre maior acúmulo da biomassa desse compartimento (BERNARDO, 1995) e devido ao espaçamento adotado, com linhas a cada $20 \mathrm{~m}$, diminui-se a quantidade de indivíduos por hectare.

Portanto, a produção de biomassa de tronco é inversamente proporcional ao espaçamento e altamente influenciada pelo número de indivíduos por hectare (OLIVEIRA NETO et al., 2003). A diminuição do espaçamento do eucalipto gera um aumento da densidade básica, influenciando consequentemente na produção de madeira (MIGLIORINI et al., 1980). 
O espaçamento influencia no crescimento das árvores e isso consequentemente afeta a produção de biomassa. Assim considerando o espaçamento e a idade, afirma-se que a produção por hectare é menor em plantios com maior espaçamento, além de que aos 77 a 85 meses tem-se uma acentuada redução da biomassa especialmente no espaçamento $3 \times 0,5 \mathrm{~m}$ e $3 \times 2 \mathrm{~m}$ (PAULINO, 2012).

A produção de biomassa também é influenciada pela capacidade produtiva do sítio e pelas condições climáticas (WANG et al., 2008). A competição intra-específica, associada a competição inter-específica entre o eucalipto e o capim Brachiaria brizantha, pode influenciar no desenvolvimento desses componentes. Para SILVA et al. (2000), as mudas de eucalipto que crescem em consórcio com gramíneas têm sua produtividade de biomassa reduzida, quando comparado ao monocultivo.

A distribuição dos nutrientes nos compartimentos da árvore é importante para a nutrição florestal, pois a cultura manejada aumenta tanto a produção de biomassa quanto a exportação de nutrientes no sítio. Observa-se que a biomassa residual (folhas + galhos finos) apresentando menor peso, possui elevado estoque de nutrientes. Andrade et al (2011) ao realizar um levantamento com 10 materiais de eucalipto verificou que os teores de alguns nutrientes na madeira e na casca podem variar expressivamente, atingindo uma variação de até 10 vezes no mesmo compartimento, dependendo do clone.

A variação no teor dos compartimentos pode também estar associado ao poder de diluição (LEITE et al., 1998) e a idade do material. $\mathrm{O}$ teor de $\mathrm{N}$ é superior no material residual, especialmente pela quantidade de folhas em comparação aos demais componentes, em virtude desse ser constituinte das estruturas responsáveis pela atividade fotossintética da planta (MALAVOLTA, 1985). Em relação aos sistemas, há diferença no teor de $\mathrm{N}$ na madeira, com o monocultivo sendo superior aos demais, resultado de árvores mais finas, menos lignificadas e de material menos denso. Schumacher (1992) registrou maiores teores de N nas folhas, os quais variaram de 19,9 a 14,0 g. $\mathrm{kg}^{-1}$ em Eucalyptus camaldulensis Dehnh, Eucalyptus grandis Hill ex Maiden, ambos com 9 anos e no Eucalyptus torelliana F. Muell, com 12 anos. Em relação ao $\mathrm{N}$ nas folhas, resultados semelhantes foram obtidos por Zaia; Gama Rodrigues (2004) estudando três espécies de eucalipto verificaram 14,9 g. $\mathrm{kg}^{-1}$ aos 72 meses.

Os teores de $\mathrm{P}$ são influenciados em praticamente todos os compartimentos, porém não há uma tendência para um cultivo ou sistema específico. Já os maiores teores de $\mathrm{K}$ no compartimento folhas e galhos finos se deve ao elemento ser móvel nos tecidos da planta, concentrando-se nas áreas ativas de crescimento e ter funções ligadas a processos vitais da planta (MARSCHNER, 1995).

Para que as árvores de eucalipto realizem suas funções vitais, elas movimentam e armazenam diferentes elementos. A quantidade estocada e a concentração dos elementos, variam nos compartimentos da árvore. Em geral, as folhas e as raízes são ricas em $\mathrm{N}$, a casca em $\mathrm{Ca}$, a madeira em $\mathrm{K}$, os galhos em Ca e K (FOELKEL, 2006).

O Ca é o elemento predominante na casca (82 a 95\%), (FENGEL; WEGENER, 1984), com teor de 0,8 a $3,5 \%$ (FOELKEL, 2006), e na sequência estão o $\mathrm{K}$ e o $\mathrm{Mg}$ (FENGEL; WEGENER, 1984). A casca armazena 45 a 55\% do total de Ca da planta. Já no caso do K, o maior acúmulo está na madeira (50 a 60\%) e na casca (15 a 20\%). Portanto, a madeira e a casca totalizam 70 a $85 \%$ de todo o $\mathrm{Ca}$ e do $\mathrm{K}$ armazenado nas árvores (FOELKEL, 2006).

$\mathrm{O}$ maior teor de $\mathrm{Mg}$ na biomassa residual $\left(5,08 \mathrm{~g} \mathrm{~kg}^{-1}\right)$ no monocultivo decorre das árvores apresentarem grande quantidade de folhas novas, devido a competição por luz. $\mathrm{O}$ $\mathrm{Mg}$ fica no centro da molécula de clorofila e participa da ativação enzimática, mais do que qualquer outro nutriente (EPSTEIN; BLOOM, 2006).

Quanto aos micronutrientes, os teores de Zn observados se assemelham aos observados por Dick et al (2017) ao avaliarem os mesmos compartimentos, contudo no caso do B, apenas nas folhas os valores se assemelham, o que pode ser devido a espécie, o tipo de cultivo, espaçamento e as condições edafoclimáticas do local de plantio.

$\mathrm{O}$ estoque total dos nutrientes foi superior no monocultivo, devido a maior densidade por hectare, mas estatisticamente semelhante ao arranjo duplo e triplo, exceto para $\mathrm{Zn}$. Ainda assim, ao considerar a densidade por hectare $(250,435,537$ e 883$)$ e o estoque nutricional de cada árvore nos diferentes sistemas, nota-se que não há a mesma ordem de grandeza $(1,7 x, 2,1 x$ e $3,3 x)$ de acúmulo total para $N, P$, $\mathrm{K}, \mathrm{S}, \mathrm{B}$ e Zn. No monocultivo tem-se um aumento de 3,3 vezes na densidade por hectare, contudo o estoque de nutrientes apenas duplica, indicando assim que os indivíduos, no arranjo de linha simples, são mais extrativos.

Para o $\mathrm{Ca}$, o estoque total no monocultivo supera em mais de uma tonelada quando comparado ao arranjo simples, resultado dos compartimentos madeira e casca, devido a maior densidade por hectare. Para $\mathrm{Mg}$, o monocultivo extraiu três vezes mais nutrientes que o arranjo simples, resultado do estoque nas folhas novas da copa das árvores. Para os demais nutrientes, o arranjo simples apresentou valores similares ou superiores ao monocultivo.

No caso específico da biomassa residual, o arranjo de linha simples, duplo e triplo apresentam estoques numericamente superiores ao monocultivo. Para $\mathrm{N}$ por exemplo, essa quantia é de mais de $50 \mathrm{~kg} \mathrm{ha}^{-1}$, resultado do grande aporte de biomassa individual de cada árvore, devido a menor competição intraespecífica, da nutrição nitrogenada mais adequada. Além disso, as árvores em consórcio com pastagem e animal reutilizam o $\mathrm{N}$ advindo do material fecal depositado pelo gado, a sombra das árvores, auxiliando assim no crescimento das mesmas.

A similaridade de exportação percentual de nutrientes para o arranjo simples versus o monocultivo demonstra que mesmo submetendo os indivíduos a ambientes diferentes, a necessidade nutricional e proporção dos elementos no tecido não apresentam muita variação. Em relação a partição de nutrientes, o $\mathrm{N}$ tem uma taxa de retorno superior a $40 \%$ no arranjo simples em relação a apenas $26 \%$ no monocultivo, sendo o macronutriente mais exportado percentualmente, seguido pelo $\mathrm{S}$, o que remete aos programas de adubação em áreas continuas com uso de floresta, com uma atenção a reposição destes elementos.

A biomassa total e o estoque de macronutrientes em plantios originários de material de propagação vegetativa é superior a plantios conduzidos por sementes. Isso é comprovado pelos valores superiores registrados pelo clone H13, do presente estudo, quando comparado aos valores registrados em um plantio seminal de E. grandis Hill exMaiden, com cinco anos (BELLOTE et al, 1980). 
Portanto, grandes quantias de macronutrientes são acumuladas e exportadas na exploração florestal (REIS; BARROS, 1990). Com a colheita da madeira e casca, tem-se uma remoção de nutrientes superior a $45 \%$ da quantia acumulada na biomassa total em um plantio de E. urophylla $\mathrm{x}$ E. globulus aos 120 meses (VIERA et al., 2015).

A variação do teor nutricional ao longo do tronco seguiu praticamente a mesma tendência verificada para Platanus $x$ acerifolia (Aiton) Willd, aos 5,5 anos (WITSCHORECK; SCHUMACHER, 2013). No entanto, a variação do teor de nutrientes na madeira e na casca ao longo do tronco não segue um padrão para os nutrientes e para as diferentes espécies (RUBILAR et al. 2005; WITSCHORECK SCHUMACHER, 2013).

\section{CONCLUSÕES}

A biomassa dos diferentes compartimentos foi superior no arranjo de linha tripla, seguido pelo arranjo de linha dupla e simples. Esses foram similares a biomassa quantificada no monocultivo de eucalipto.

$\mathrm{O}$ maior teor de nutrientes ocorre na biomassa residual e na madeira. No entanto, a biomassa residual e a casca correspondem a uma fração expressiva de nutrientes que permanece no sistema, e que contribuem para menor reposição na manutenção de nutrientes após colheita.

$\mathrm{Na}$ biomassa residual e na casca, o estoque de $\mathrm{N}, \mathrm{Ca}$ e o $\mathrm{K}$ são mais expressivos, sendo que para os galhos grossos, apenas o $\mathrm{N}$ e o $\mathrm{K}$ apresentam estoque elevado, para todos os arranjos de plantio.

$\mathrm{O}$ arranjo de plantio das árvores não influencia no teor de nutrientes da madeira ao longo da posição do tronco das árvores, exceto para os teores nutricionais na casca.

\section{AGRADECIMENTOS}

Á Embrapa Agrossilvipastoril pelo apoio em disponibilizar a área para o estudo.

\section{REFERÊNCIAS}

ALVARES, C. A.; STAPE, J. L., SENTELHAS, P. C.; GONÇALVEZ, J. L. M.; SPAROVEK, G. Köppen's climate classification map for Brazil. Meteorologische Zeitschrift, Berlin, v. 22, n. 6, p. 711-728. DOI: DOI: https://dx.doi.org/10.1127/0941-2948/2013/0507

ADRANDE, M. C. de; MINHONI, M. T. de A.; SANSÍGOLO, C. A.; ZIED, D. C.; SALES-CAMPOS, C. Estudo comparativo da constituição nutricional da madeira e casca de espécies e clones de eucalipto visando o cultivo de shiitake em toras. Revista Árvore, Viçosa, n. 35, n. 2, p. 183-192, 2011. DOI: http://dx.doi.org/10.1590/S0100-67622011000200002

BAGGIO, A. J.; CARPANEZZI, A. A. Biomassa aérea da bracatinga Mimosa scabrella Benth. em talhões do sistema de cultivo tradicional. Boletim de Pesquisa Florestal, Colombo, n. 34, p. 31-44, 1997.

BALBINO, L. C.; CORDEIRO, L. A. M.; SILVA, V.P.; MORAES, A.; MARTÍNEZ, G. B.; ALVARENGA, R. C.; KICHEL, A. N.; FONTANELI, R. S.; SANTOS, H. P.; FRANCHINI, J. C.; GALERAN, P. R. Evolução Tecnológica e Arranjos Produtivos de Sistemas de Integração Lavoura-Pecuária-Floresta no Brasil. Pesquisa Agropecuária Brasileira, Brasília, v. 46, n. 10, 2011. DOI: 204X2011001000001
BELlOTE, A. F. J.; SARRUGE, J. R.; HAAG, H. P.; OLIVEIRA, G. D. de. Extração e exportação de nutriente pelo Eucalyptus grandis Hill ex-Maiden em função da idade: macronutrientes. Scientia Forestalis, Piracicaba, n. 20, p. 27-45, 1980.

BERNARDO, A. L. Crescimento e eficiência nutricional de Eucalyptusssp. sob diferentes espaçamentos na região do cerrado de Minas Gerais. 1995. $102 \mathrm{f}$. Dissertação (Mestrado em Ciências Florestais) Universidade Federal de Viçosa, Viçosa, 1995.

CARVALHO, G. Produtividade, partição de biomassa e nutrients em sistemas de integração lavoura-pecuáriafloresta. 2017. 186f. Dissertação (Mestrado em Agronomia) - Universidade Federal de Mato Grosso, Sinop, 2017.

COELHO JÚNIOR, J.M.L.P. Biomassa e volumetria de híbridos de Eucalyptus Urograndis em sistema de integração lavoura-pecuária floresta (ILPF) na região sul de Goiás. 2015. 63f. Dissertação (Mestrado em Agronomia) - Universidade Federal de Goiás, Goiânia, 2015.

DICK, G.; SCHUMACHER, M. V.; MOMOLLI, D. R.; GUIMARÃES, C. de C.; SOUZA, H. P. LUDVICHAK, A. A. Micronutrients and biomass in Eucalyptus dunnii Maiden Stand. Revista Árvore, Viçosa, v. 41, n. 1, e410113, 2017. DOI: http://dx.doi.org/10.1590/180690882017000100013

EPSTEIN, E.; BLOOM, A. J. Nutrição Mineral de plantas: princípios e perspectivas. Tradução Maria Edna Tenório Nunes. 2. ed. Londrina: Editora Planta, 2006. 403 p.

FENGEL, D.; WEGENER, G. Wood, chemistry, ultrastructure, reactions. New York: Walter de Gruyter, $1984.613 \mathrm{p}$.

FOELKEL, C. Casca da árvore do eucalipto: aspectos morfológicos, fisiológicos, florestais, ecológicos e industriais, visando a produção de celulose e papel. Eucalyptus Book \& Newsletter, 2006. 109p. Disponível em:

$<$ http://www.eucalyptus.com.br/capitulos/capitulo_casca. pdf $>$ Acesso em: 10 de novembro de 2016.

LEITE, F. P.; BARROS, N. F.; NOVAIS, R. F.; FABRES, A. S. Acúmulo e distribuição de nutrientes em Eucalyptus grandis sob diferentes densidades populacionais. Revista Brasileira de Ciência do Solo, Viçosa, v. 22, n. 3, p. 419-426, 1998. DOI: http://dx.doi.org/10.1590/S010006831998000300007

MALAVOLTA, E. Absorção e transporte de íons e nutrição mineral. In: FERRI, M. G. Fisiologia vegetal. 1. ed. São Paulo: EPU, 1985. p. 77-116.

MARSCHNER, H. Mineral nutrition of higher plants. 2. ed. London: Academic Press, 1995. 889 p.

MIGLIORINI, A. J.; BRITO, J. O.; BARRICHELO, L. E. G. Influência das práticas silviculturais na produção de carvão vegetal. Piracicaba: IPEF, n. 104, 1980. (CIRCULAR TÉCNICA, 104)

MÜLLER, M. D.; TSUKAMOTO FILHO, A. A.; VALE, R. S. do; COUTO, L. Produção de biomassa e conteúdo energético em sistemas agroflorestais com Eucalipto, no Município de Vazendo, MG. Biomassa e Energia, Viçosa, v. 2, n. 1, p. 125-132, jan./mar. 2005

OLIVEIRA NETO, S. N. de; REIS, G. G. dos; REIS, M. G. F.; NEVES, J. C. L. Produção e distribuição de biomassa em Eucalyptus camaldulensis Dehn. em resposta à 
adubação e ao espaçamento. Revista Árvore, Viçosa, v. 27, n. 1, p. 15-23, 2003. DOI: http://dx.doi.org/10.1590/S0100-67622003000100003

PAULINO, E. J. Influência do espaçamento e da idade na produção de biomassa e na rotação econômica em plantios de eucalipto. 2012. 60 p. Dissertação (Mestrado em Ciência Florestal) - Universidade Federal dos Vales do Jequitinhonha e Mucuri, Diamantina, 2012.

REIS, M. G. F.; BARROS, N. F. Ciclagem de nutrientes em plantio de eucalipto. In: BARROS, N. F.; NOVAIS, R. F. (Ed.). Relação solo-eucalipto. Viçosa: Editora Folha de Viçosa, 1990. p. 265-301.

RUBILAR, R. A.; ALlEN, H. L.; KELtinG, D. L. Comparison of biomass and nutrient content equations for successive rotations of loblolly pine plantations on an Upper Coastal Plain Site. Biomass and Bioenergy, Oxford, v. 28, n. 6, p. 548-564, 2005. DOI: https://dx.doi.org/10.1016/j.biombioe.2004.12.001

SANTOS, H. G. dos; JACOMINE, P. K. T.; ANJOS, L. H. C. dos; OLIVEIRA, V. A. de; LUMBRERAS, J. F.; COELHO, M. R.; ALMEIDA, J. A. de; ARAUJO FILHO, J. C. de; OLIVEIRA, J. B. de; CUNHA, T. J. F. Sistema Brasileiro de Classificação de Solos. 3. ed. rev. e ampl. Brasília: Embrapa, 2013.353 p.

SCHUMACHER, M. V. Aspectos da ciclagem de nutrientes e do microclima em talhões de Eucalyptus camaldulensis Dehnh, Eucalyptus grandis Hill ex Maiden e Eucalyptus torrelliana F. Mesell. 1992. 87f. Dissertação (Mestrado em Ciências) - Escola Superior de Agricultura "Luiz de Queiroz", Piracicaba, 1992. plantas e fertilizantes. 2. ed. Brasília: Embrapa Informação Tecnológica, 2009. 627 p.

SILVA, W.; SILVA, A. A.; SEDIYAMA, T.; FREITAS, R. S. Absorção de nutrientes por mudas de duas espécies de eucalipto em resposta a diferentes teores de água no solo e competição com plantas de Brachiaria brizantha. Ciências Agrotecnicas, Lavras, v. 24, n. 1, p. 147-159, 2000.

TSUKAMOTO FILHO, A. A.; COUTO, L.; NEVES, J. C. L.; PASSOS, C. A. M.; SILVA, M. L. Fixação do carbono em um sistema agrosilvipastoril com eucalipto na região do Cerrado em Minas Gerais. Agrossilvicultura, v. 1, n. 1, p. 29-41, 2004.

VIERA, M.; SCHUMACHER, M. V.; TRÜBY, P.; ARAÚJO, E. F. Implicações nutricionais com base em diferentes intensidades de colheita da biomassa de Eucalyptus urophylla x Eucalyptus globulus. Ciência Rural, Santa Maria, v. 45, n. 3, p. 432-439, mar. 2015. DOI: http://dx.doi.org/10.1590/0103-8478cr20120367

WANG, X.; FANG, J.; ZHU, B. Forest biomass and rootshoot allocation in northeast China. Forest Ecology and Management, Amsterdam, v. 255, n. 12, p. 4007-4020, 2008.

DOI: https://dx.doi.org/10.1016/j.foreco.2008.03.055

WITSCHORECK, R.; SCHUMACHER, M. V. Teor e alocação de nutrientes em plantio de Platanus x acerifolia (Aiton) Willd. em Dom Feliciano - RS. Ciência Florestal, Santa Maria, v. 23, n. 4, p. 667-678, out-dez, 2013. DOI: http://dx.doi.org/10.5902/1980509810538

ZAIA, F. C.; GAMA-RODRIGUES, A. C. Ciclagem e balanço de nutrientes em povoamentos de eucalipto na região norte fluminense. Revista Brasileira de Ciência do Solo, Viçosa, v. 28, n. 5, p. 843-852, 2004. DOI: http://dx.doi.org/10.1590/S0100-06832004000500007 